\title{
Iterative approximation of fixed points of Prešić operators on partial metric spaces
}

\author{
M. Abbas ${ }^{1}$, M. Berzig ${ }^{2}$, T. Nazir ${ }^{3}$ and E. Karapınar ${ }^{4}$
}

\begin{abstract}
We study the convergence of the Prešić type $k$-step iterative process for a class of operators $f: X^{k} \rightarrow X$ satisfying Prešić type F-contractive condition in the setting of metric spaces. As an applications of the result presented herein, we derive global attractivity results for a class of matrix difference equations. Numerical experiments are also presented to illustrate the theoretical findings.
\end{abstract}

Keywords: Fixed point approximation, iterative method, $F$-contraction, equilibrium point, matrix difference equation, global attractivity

MSC2010: 47H10, 54H25, 65Q10,65Q30

\section{Introduction and Preliminaries}

Banach contraction mapping principle [?] is simple and powerful result with a wide range of applications, including iterative methods for solving linear, nonlinear, differential, integral, and difference equations. There are several generalizations and extensions of the Banach contraction principle in the existing literature. Recently, Wardowski [?] introduced a new class of contractions called $F$-contraction and proved a fixed point result as a generalization of the Banach contraction principle [?].

We begin with some basic known definitions and results which will be used in the sequel. Throughout this article, $\mathbb{N}, \mathbb{R}^{+}, \mathbb{R}$ denote the set of natural numbers, the set of positive real numbers and the set of real numbers, respectively.

Let $\digamma$ be the collection of all mappings $F: \mathbb{R}^{+} \rightarrow \mathbb{R}$ that satisfy the following conditions:

$\left(F_{1}\right) F$ is strictly increasing, that is, for all $\alpha, \beta \in \mathbb{R}^{+}$such that $\alpha<\beta$ implies that $F(\alpha)<F(\beta)$.

\footnotetext{
${ }^{1}$ Department of Mathematics and Applied Mathematics University of Pretoria, Pretoria 0002, South Africa, E-mail: mujahid.abbas@up.ac.za

${ }^{2}$ Université de Tunis, Ecole Supérieure des Sciences et Techniques de Tunis, Bab Menara, Tunisia, E-mail: berzig@gmail.com

${ }^{3}$ Department of Mathematics, COMSATS Institute of Information Technology, Abbottabad 22060, Pakistan and Department of Mathematics, Walter Sisulu University, Mthatha 5117, South Africa, E-mail: talat@ciit.net.pk

${ }^{4}$ Department of Mathematics, Atılım University, Incek, Ankara, 06836, Turkey, E-mail: erdalkarapinar@yahoo.com. Second address: Nonlinear Analysis and Applied Mathematics Research Group (NAAM), King Abdulaziz University, Jeddah, Saudi Arabia.
} 
$\left(F_{2}\right)$ For every sequence $\left\{\alpha_{n}\right\}$ of positive real numbers, $\lim _{n \rightarrow \infty} \alpha_{n}=0$ and $\lim _{n \rightarrow \infty} F\left(\alpha_{n}\right)=$ $-\infty$ are equivalent.

$\left(F_{3}\right)$ There exists $h \in(0,1)$ such that $\lim _{\alpha \rightarrow 0^{+}} \alpha^{h} F(\alpha)=0$.

Definition 1.1. [23] Let $(X, d)$ be a metric space and $F \in \digamma$. A mapping $f: X \rightarrow X$ is said to be an $F$-contraction on $X$ if there exists $\tau>0$ such that

$$
d(f x, f y)>0 \text { implies that } \tau+F(d(f x, f y)) \leq F(d(x, y))
$$

for all $x, y \in X$.

Note that every $F$-contraction is continuous (see [23]).

Wardowski obtained the following result.

Theorem 1.1. Let $(X, d)$ be a complete metric space and $f: X \rightarrow X$ an $F$-contraction. Then there exists a unique $x$ in $X$ such that $x=f x$. Moreover, for any $x_{0} \in X$, the iterative sequence $\left\{x_{n}\right\}$ defined by $x_{n+1}=f\left(x_{n}\right)$ converges to $x$

Let $f: X^{k} \rightarrow X$, where $k \geq 1$ is a positive integer. A point $x^{*} \in X$ is called a fixed point of $f$ if $x^{*}=f\left(x^{*}, \ldots, x^{*}\right)$.

Consider the $k$-th order nonlinear difference equation:

$$
x_{n+k}=f\left(x_{n}, x_{n+1}, \ldots, x_{n+k-1}\right), n=1,2, \ldots
$$

with the initial values $x_{1}, \ldots, x_{k} \in X$.

Equation (1) can be studied by means of fixed point theory in view of the fact that $x$ in $X$ is a solution of (1) if and only if $x$ is a fixed point of mapping $T: X \rightarrow X$ given by

$$
T(x)=f(x, x, \ldots, x) \text {, for all } x \in X .
$$

One of the most important results in this direction is due to Prešić [16] given as follows:

Theorem 1.2. [16] Let $(X, d)$ be a complete metric space, $k$ a positive integer. If a mapping $f: X^{k} \rightarrow X$ satisfies the following contractive condition:

$$
d\left(f\left(x_{1}, x_{2}, \ldots, x_{k}\right), f\left(x_{2}, \ldots, x_{k}, x_{k+1}\right)\right) \leq q_{1} d\left(x_{1}, x_{2}\right)+q_{2} d\left(x_{2}, x_{3}\right)+\ldots+q_{k} d\left(x_{k}, x_{k+1}\right),
$$

for every $x_{1}, \ldots, x_{k+1} \in X$, where $q_{1}, q_{2}, \ldots, q_{k}$ are non-negative constants such that $q_{1}+$ $q_{2}+\ldots+q_{k}<1$. Then there exists a unique point $x^{*} \in X$ such that $f\left(x^{*}, \ldots, x^{*}\right)=x^{*}$. Moreover, for any arbitrary points $x_{1}, \ldots, x_{k} \in X$, the sequence (1) converges to $x^{*}$.

Note that, for $k=1$, Theorem 1.2 reduces to the Banach contraction principle.

Ćirić and Prešić [7] generalized the above result as follows.

Theorem 1.3. [7] Let $(X, d)$ be a complete metric space, and $k$ a positive integer. If $f: X^{k} \rightarrow X$ satisfies the following contractive condition:

$$
d\left(f\left(x_{1}, x_{2}, \ldots, x_{k}\right), f\left(x_{2}, \ldots, x_{k}, x_{k+1}\right)\right) \leq q \max \left\{d\left(x_{1}, x_{2}\right), d\left(x_{2}, x_{3}\right), \ldots, d\left(x_{k}, x_{k+1}\right)\right\},
$$

for any $x_{1}, \ldots, x_{k+1} \in X$, where $0<q<1$. Then there exists $x^{*} \in X$ such that $f\left(x^{*}, \ldots, x^{*}\right)=x^{*}$. Moreover, for any arbitrary points $x_{1}, \ldots, x_{k} \in X$, the sequence (1.1) is convergent and

$$
\lim _{n \rightarrow \infty} x_{n}=f\left(\lim _{n \rightarrow \infty} x_{n}, \ldots, \lim _{n \rightarrow \infty} x_{n}\right) .
$$


If in addition,

$$
d(T(u, \ldots, u), T(v, \ldots, v))<d(u, v)
$$

holds for all $u, v \in X$, with $u \neq v$, then $x^{*}$ is the unique point in $X$ with $f\left(x^{*}, \ldots, x^{*}\right)=x^{*}$.

The applicability of the above result to the study of global asymptotic stability of the equilibrium for the nonlinear difference equation (1) is well known ([5]).

Păcurar [15] derived a convergence result for Prešić-Kannan operators as follows:

Theorem 1.4. [15] Let $(X, d)$ be a complete metric space, $k$ a positive integer and $f$ : $X^{k} \rightarrow X$ a given mapping. Suppose that there exists a constant $a \in \mathbb{R}$ with $0<a k(k+1)<$ 1 such that

$$
d\left(f\left(x_{1}, \ldots, x_{k}\right), f\left(x_{2}, \ldots, x_{k+1}\right)\right) \leq a \sum_{i=1}^{k+1} d\left(x_{i}, f\left(x_{i}, \ldots, x_{i}\right)\right),
$$

holds for all $\left(x_{1}, \ldots, x_{k+1}\right) \in X^{k+1}$. Then,

(i) $f$ has a unique fixed point $x^{*} \in X$;

(ii) for any arbitrary points $x_{1}, \ldots, x_{k} \in X$, the sequence $\left\{x_{n}\right\}$ defined by (1) converges to $x^{*}$.

For more results in this direction, we refer to $[2,3,5,7,9,10,11,14,15,17,18,19$, $20,21]$.

Motivated by the work of Wardowski [23], we give the following definition:

Definition 1.2. Let $(X, d)$ be a metric space and $F \in \digamma$. A mapping $f: X^{k} \rightarrow X$ is said to be a Prešić type $F$-contraction if there exists $\tau>0$ such that

$$
d\left(f\left(x_{1}, \ldots, x_{k}\right), f\left(x_{2}, \ldots, x_{k+1}\right)\right)>0
$$

implies that

$$
\tau+F\left(d\left(f\left(x_{1}, \ldots, x_{k}\right), f\left(x_{2}, \ldots, x_{k+1}\right)\right)\right) \leq F\left(\max \left\{d\left(x_{i}, x_{i+1}\right): 1 \leq i \leq k\right\}\right)
$$

for all $\left(x_{1}, \ldots, x_{k+1}\right) \in X^{k+1}$.

Note that, for $F(\alpha)=\ln \alpha$, Prešić type $F$-contraction condition becomes

$$
d\left(f\left(x_{1}, \ldots, x_{k}\right), f\left(x_{2}, \ldots, x_{k+1}\right)\right) \leq e^{-\tau} \max \left\{d\left(x_{i}, x_{i+1}\right): 1 \leq i \leq k\right\}
$$

for all $\left(x_{1}, \ldots, x_{k+1}\right) \in X^{k+1}, f\left(x_{1}, \ldots, x_{k}\right) \neq f\left(x_{2}, \ldots, x_{k+1}\right)$.

Furthermore, for $\left(x_{1}, \ldots, x_{k+1}\right) \in X^{k+1}$ such that $f\left(x_{1}, \ldots, x_{k}\right)=f\left(x_{2}, \ldots, x_{k+1}\right)$, the inequality (3) also holds, that is, $f$ is a Ćirić-Prešić contraction (see [7]).

Remark 1.1. It follows from $\left(F_{1}\right)$ and $(2)$ that every Prešić type $F$-contraction mapping $f$ is a Prešic contractive mapping, that is,

$$
d\left(f\left(x_{1}, \ldots, x_{k}\right), f\left(x_{2}, \ldots, x_{k+1}\right)\right)<\max \left\{d\left(x_{i}, x_{i+1}\right): 1 \leq i \leq k\right\}
$$

for all $\left(x_{1}, \ldots, x_{k+1}\right) \in X^{k+1}, f\left(x_{1}, \ldots, x_{k}\right) \neq f\left(x_{2}, \ldots, x_{k+1}\right)$. 
Thus every Prešić type $F$-contraction mapping $f$ is a continuous mapping.

The aim of this paper is to study the convergence of the sequence $\left\{x_{n}\right\}$ defined by (1), where $f: X^{k} \rightarrow X$ is a Prešić type $F$ - contraction mapping. We also give examples to support the results presented herein. Applications to matrix difference equations are also presented.

\section{Main results}

In this section, we obtain some fixed point results for Prešić type $F$-contraction mappings. We start with the following result.

Theorem 2.1. Let $(X, d)$ be a complete metric space and $f: X^{k} \rightarrow X$ a Prešić type $F$-contraction, where $k$ is a positive integer. Then, for any arbitrary points $x_{1}, \ldots, x_{k} \in X$, the sequence $\left\{x_{n}\right\}$ defined by (1) converges to $u$ in $X$ and $u$ is a fixed point of $f$. In addition, if

$$
d(f(x, \ldots, x), f(y, \ldots, y))>0
$$

implies that

$$
\tau+F(d(f(x, \ldots, x), f(y, \ldots, y))) \leq F(d(x, y))
$$

for all $x, y \in X$ with $x \neq y$, then $f$ has a unique fixed point.

Proof. First, let us observe that $f$ has at most one fixed point. Indeed, if $u, v \in X$ such that $u=f(u, \ldots, u)$ and $v=f(v, \ldots, v)$ with $u \neq v$. Thus $d(f(u, \ldots, u), f(v, \ldots, v))>0$. Hence by given assumption, we have

$$
\begin{aligned}
\tau+F(d(u, v)) & =\tau+F(d(f(u, \ldots, u), f(v, \ldots, v))) \\
& \leq F(d(u, v)),
\end{aligned}
$$

a contradiction as $\tau>0$. Therefore $u=v$.

Now we show that $f$ has a fixed point. Let $x_{1}, \cdots, x_{k}$ be arbitrary $k$ elements in $X$. Define the sequence $\left\{x_{n}\right\}$ in $X$ by

$$
x_{n+k}=f\left(x_{n}, x_{n+1}, \ldots, x_{n+k-1}\right), n=1,2, \ldots .
$$

If for some $n_{0} \in\{1,2,3, \ldots, k\}$, we have $x_{n_{0}}=x_{n_{0}+1}$, then we have

$$
x_{n_{0}+k}=f\left(x_{n_{0}}, x_{n_{0}+1}, \ldots, x_{n_{0}+k-1}\right)=f\left(x_{n_{0}+k}, x_{n_{0}+k}, \ldots, x_{n_{0}+k}\right),
$$

that is, $x_{n_{0}+k}$ is a fixed point of $f$ and the proof is finished.

We assume that $x_{n+k} \neq x_{n+k+1}$ for all $n \in \mathbb{N}$. Denote $\gamma_{n+k}=d\left(x_{n+k}, x_{n+k+1}\right)$ for $n=1,2, \ldots$ and $\theta=\max \left\{d\left(x_{1}, x_{2}\right), d\left(x_{2}, x_{3}\right), \ldots, d\left(x_{k}, x_{k+1}\right)\right\}$, then we have $\gamma_{n+k}>0$ for all $n \in \mathbb{N}$ and $\theta>0$.

Now for $n \leq k$, we have the following inequalities:

$$
\begin{aligned}
F\left(\gamma_{k+1}\right) & =F\left(d\left(x_{k+1}, x_{k+2}\right)\right) \\
& =F\left(d\left(f\left(x_{1}, x_{2}, \ldots, x_{k}\right), f\left(x_{2}, x_{3}, \ldots, x_{k+1}\right)\right)\right) \\
& \leq F\left(\max \left\{d\left(x_{i}, x_{i+1}\right): 1 \leq i \leq k\right\}\right)-\tau \\
& =F(\theta)-\tau
\end{aligned}
$$




$$
\begin{aligned}
F\left(\gamma_{k+2}\right) & =F\left(d\left(x_{k+2}, x_{k+3}\right)\right) \\
& =F\left(d\left(f\left(x_{2}, x_{3}, \ldots, x_{k+1}\right), f\left(x_{3}, x_{4}, \ldots, x_{k+2}\right)\right)\right) \\
& \leq F\left(\max \left\{d\left(x_{i}, x_{i+1}\right): 2 \leq i \leq k+1\right\}\right)-\tau \\
& \leq F(\theta)-2 \tau
\end{aligned}
$$

and so on. Hence

$$
\begin{aligned}
F\left(\gamma_{k+n}\right) & =F\left(d\left(x_{n+k}, x_{n+k+1}\right)\right) \\
& =F\left(d\left(f\left(x_{n}, x_{n+1}, \ldots, x_{n+k-1}\right), f\left(x_{n+1}, x_{n+2}, \ldots, x_{n+k}\right)\right)\right) \\
& \leq F(\theta)-n \tau
\end{aligned}
$$

for $n \geq 1$. On taking limit as $n \rightarrow \infty$, we obtain that $\lim _{n \rightarrow \infty} F\left(\gamma_{k+n}\right)=-\infty$ and therefore $\lim _{n \rightarrow \infty} \gamma_{k+n}=0$ by $\left(F_{2}\right)$.

Now by $\left(F_{3}\right)$, there exists $h \in(0,1)$ such that

$$
\lim _{n \rightarrow \infty} \gamma_{k+n}^{h} F\left(\gamma_{k+n}\right)=0
$$

By (4), we have

$$
\gamma_{k+n}^{h} F\left(\gamma_{k+n}\right)-\gamma_{k+n}^{h} F(\theta) \leq \gamma_{k+n}^{h}(F(\theta)-n \tau)-\gamma_{k+n}^{h} F(\theta)=-\gamma_{k+n}^{h} n \tau \leq 0 .
$$

On taking limit as $n \rightarrow \infty$, we obtain

$$
\lim _{n \rightarrow \infty} n \gamma_{k+n}^{h}=0
$$

Thus from (4), there exists $n_{0} \in \mathbb{N}$ such that $n \gamma_{k+n}^{h} \leq 1$ for all $n \geq n_{0}$. Consequently we have

$$
\gamma_{k+n} \leq \frac{1}{n^{1 / h}} \text { for all } n \geq n_{0}
$$

For any $n, m \in \mathbb{N}$ with $m \geq n \geq n_{0}$, we have

$$
\begin{aligned}
d\left(x_{k+n}, x_{k+m}\right)= & d\left(f\left(x_{n}, \ldots, x_{k+n-1}\right), f\left(x_{m}, \ldots, x_{k+m-1}\right)\right) \\
\leq & d\left(f\left(x_{n}, \ldots, x_{k+n-1}\right), f\left(x_{n+1}, \ldots, x_{k+n}\right)\right)+d\left(f\left(x_{n+1}, \ldots, x_{k+n}\right), f\left(x_{n+2}, \ldots, x_{k+n+1}\right)\right) \\
& +\ldots+d\left(f\left(x_{m-1}, \ldots, x_{k+m-2}\right), f\left(x_{m}, \ldots, x_{k+m-1}\right)\right) \\
= & d\left(x_{n+k}, x_{n+k+1}\right)+d\left(x_{n+k+1}, x_{n+k+2}\right)+\ldots+d\left(x_{m+k-1}, x_{m+k}\right) \\
= & \gamma_{n+k}+\gamma_{n+k+1}+\ldots+\gamma_{m+k-1} \\
< & \sum_{i=n}^{\infty} \gamma_{i+k} \leq \sum_{i=n}^{\infty} \frac{1}{i^{1 / h}} \rightarrow 0
\end{aligned}
$$

This shows that $\left\{x_{n}\right\}$ is a Cauchy sequence in $(X, d)$. Since $(X, d)$ is complete, there exists $u$ in $X$ such that

$$
\lim _{n, m \rightarrow \infty} d\left(x_{n}, x_{m}\right)=\lim _{n \rightarrow \infty} d\left(x_{n}, u\right)=0
$$


Now by the continuity of $f$, we have

$$
\begin{aligned}
u & =\lim _{n \rightarrow \infty} x_{n+k}=\lim _{n \rightarrow \infty} f\left(x_{n}, x_{n+1}, \ldots, x_{n+k-1}\right) \\
& =f\left(\lim _{n \rightarrow \infty} x_{n}, \lim _{n \rightarrow \infty} x_{n+1}, \ldots, \lim _{n \rightarrow \infty} x_{n+k-1}\right) \\
& =f(u, u, \ldots, u) .
\end{aligned}
$$

Example 2.1. Let $X=\left\{x_{n}=\frac{n(n+1)}{2}: n \in \mathbb{N}\right\}$ and $d(x, y)=|x-y|$. Then $(X, d)$ is a complete metric space. Define the mapping $f: X^{2} \rightarrow X$ by

$$
f(x, y)= \begin{cases}\frac{x_{n-1}+y_{m-1}}{2}, & \text { if } x=x_{n}, y=y_{m} \text { for } n, m>1, \\ \frac{x_{1}+y_{1}}{2}, & \text { otherwise. }\end{cases}
$$

Note that for $n>3$, we have

$$
\begin{aligned}
& d\left(f\left(x_{n-2}, x_{n-1}\right), f\left(x_{n-1}, x_{n}\right)\right) \\
= & \frac{1}{4}\left|\left(\frac{(n-3)(n-2)}{2}+\frac{(n-2)(n-1)}{2}\right)-\left(\frac{(n-2)(n-1)}{2}+\frac{(n-1) n}{2}\right)\right| \\
= & \frac{1}{4}(4 n-6)=n-\frac{3}{2}
\end{aligned}
$$

and

$$
\begin{aligned}
& \max \left\{d\left(x_{n-2}, x_{n-1}\right), d\left(x_{n-1}, x_{n}\right)\right\} \\
= & \max \left\{\left|\frac{(n-2)(n-1)}{2}-\frac{(n-1) n}{2}\right|,\left|\frac{n(n-1)}{2}-\frac{n(n+1)}{2}\right|\right\} \\
= & \max \{n-1, n\}=n .
\end{aligned}
$$

Now

$$
\lim _{n \rightarrow \infty} \frac{d\left(f\left(x_{n-2}, x_{n-1}\right), f\left(x_{n-1}, x_{n}\right)\right)}{\max \left\{d\left(x_{n-2}, x_{n-1}\right), d\left(x_{n-1}, x_{n}\right)\right\}}=\lim _{n \rightarrow \infty} \frac{n-3 / 2}{n}=1
$$

Thus

$$
d\left(f\left(x_{n-2}, x_{n-1}\right), f\left(x_{n-1}, x_{n}\right)\right) \leq q \max \left\{d\left(x_{n-2}, x_{n-1}\right), d\left(x_{n-1}, x_{n}\right)\right\}
$$

does not hold for $q \in(0,1)$. Hence the condition of Theorem 2 in [7] is not satisfied.

On the other hand by taking $F(\alpha)=\ln \alpha+\alpha, \alpha>0$ and $\tau=1$, for $f\left(x_{i}, x_{i+1}\right) \neq$ $f\left(x_{i+1}, x_{i+2}\right) i=1,2, \ldots$, . We consider the following cases:

(1) If $x=x_{1}, y=x_{2}$, then

$$
\begin{aligned}
& d\left(f\left(x_{1}, x_{2}\right), f\left(x_{2}, x_{3}\right)\right) e^{d\left(f\left(x_{1}, x_{2}\right), f\left(x_{2}, x_{3}\right)\right)-\max \left\{d\left(x_{1}, x_{2}\right), d\left(x_{2}, x_{3}\right)\right\}} \\
= & |1-2| e^{|1-2|-3} \\
= & e^{-2}<3 e^{-1}=e^{-1} \max \left\{d\left(x_{1}, x_{2}\right), d\left(x_{2}, x_{3}\right)\right\} .
\end{aligned}
$$


(2) For $x=x_{n}, y=x_{n+1}$ with $n>1$, we have

$$
\begin{aligned}
& d\left(f\left(x_{n}, x_{n+1}\right), f\left(x_{n+1}, x_{n+2}\right)\right) e^{d\left(f\left(x_{n}, x_{n+1}\right), f\left(x_{n+1}, x_{n+2}\right)\right)-\max \left\{d\left(x_{n}, x_{n+1}\right), d\left(x_{n+1}, x_{n+2}\right)\right\}} \\
= & \left(n+\frac{1}{2}\right) e^{\left(n+\frac{1}{2}\right)-(n+2)}=\left(n+\frac{1}{2}\right) e^{-\frac{3}{2}} \\
< & e^{-1}(n+2)=e^{-1} \max \left\{d\left(x_{n}, x_{n+1}\right), d\left(x_{n+1}, x_{n+2}\right)\right\} .
\end{aligned}
$$

Thus $f$ is the Prešic type $F$-contraction on $X$ and $(1,1)$ is a unique fixed point of $f$, that is, $f(1,1)=1$.

Example 2.2. Let $X=[0,2]$ and $d$ be a usual metric of $X$. Let $k$ be a positive integer and $f: X^{k} \rightarrow X$ be the mapping defined by

$$
f\left(x_{1}, \ldots, x_{k}\right)=\frac{x_{1}+x_{k}}{4 k} \text { for all } x_{1}, \ldots, x_{k} \in X .
$$

Define $F: \mathbb{R}^{+} \rightarrow \mathbb{R}$ by $F(\alpha)=\alpha+\ln (\alpha)$. Note that $F \in \digamma \quad([23])$.

Note that, for $\tau=\ln (2 k)>0$ and $x_{1}, x_{2}, \ldots, x_{k+1} \in X$, with

$$
d\left(f\left(x_{1}, \ldots, x_{k}\right), f\left(x_{2}, \ldots, x_{k+1}\right)\right)>0,
$$

we have

$$
\begin{aligned}
& \tau+F\left(d\left(f\left(x_{1}, \ldots, x_{k}\right), f\left(x_{2}, \ldots, x_{k+1}\right)\right)\right) \\
= & \ln (2 k)+F\left(\left|\frac{x_{1}+x_{k}}{4 k}-\frac{x_{2}+x_{k+1}}{4 k}\right|\right) \\
= & \ln (2 k)+F\left(\frac{1}{4 k}\left|\left(x_{1}-x_{2}\right)+\left(x_{k}-x_{k+1}\right)\right|\right) \\
= & \ln (2 k)+\frac{1}{4 k}\left|\left(x_{1}-x_{2}\right)+\left(x_{k}-x_{k+1}\right)\right|+\ln \left(\frac{1}{4 k}\left|\left(x_{1}-x_{2}\right)+\left(x_{k}-x_{k+1}\right)\right|\right) \\
\leq & \ln (2 k)+\frac{1}{2 k} \max \left\{d\left(x_{1}, x_{2}\right), d\left(x_{k}, x_{k+1}\right)\right\}+\ln \left(\frac{1}{2 k} \max \left\{d\left(x_{1}, x_{2}\right), d\left(x_{k}, x_{k+1}\right)\right\}\right) \\
= & \frac{1}{2 k} \max \left\{d\left(x_{1}, x_{2}\right), d\left(x_{k}, x_{k+1}\right)\right\}+\ln \left(\max \left\{d\left(x_{1}, x_{2}\right), d\left(x_{k}, x_{k+1}\right)\right\}\right) \\
\leq & \max \left\{d\left(x_{i}, x_{i+1}\right): 1 \leq i \leq k\right\}+\ln \left(\max \left\{d\left(x_{i}, x_{i+1}\right): 1 \leq i \leq k\right\}\right) \\
= & F\left(\max \left\{d\left(x_{i}, x_{i+1}\right): 1 \leq i \leq k\right\}\right) .
\end{aligned}
$$

Moreover, for all $x, y \in X$ with $x \neq y$, we have

$$
d(f(x, \ldots, x), f(y, \ldots, y))=\frac{|x-y|}{2 k}>0
$$


and

$$
\begin{aligned}
F(d(f(x, \ldots, x), f(y, \ldots, y))) & =F\left(\frac{|x-y|}{2 k}\right) \\
& =\frac{|x-y|}{2 k}+\ln \left(\frac{|x-y|}{2 k}\right) \\
& =\frac{1}{2 k} d(x, y)+\ln (d(x, y))-\ln (2 k) \\
& \leq d(x, y)+\ln (d(x, y))-\ln (2 k) \\
& =F(d(x, y))-\tau .
\end{aligned}
$$

Thus, all the required hypotheses of Theorem 2.1 are satisfied. Moreover, for any arbitrary points $x_{1}, \ldots, x_{k} \in X$, the sequence $\left\{x_{n}\right\}$ defined by (1) converges to $u=0$, the unique fixed point of $f$. $\ln \alpha$.

The following result is an immediate consequence of Theorem 2.1 by taking $F(\alpha)=$

Corollary 2.1. Let $(X, d)$ be a complete metric space, $k$ a positive integer and $f: X^{k} \rightarrow X$ a given mapping. Suppose that there exists $\tau>0$ such that

$$
d\left(f\left(x_{1}, \ldots, x_{k}\right), f\left(x_{2}, \ldots, x_{k+1}\right)\right) \leq e^{-\tau} \max \left\{d\left(x_{i}, x_{i+1}\right): 1 \leq i \leq k\right\},
$$

for all $\left(x_{1}, \ldots, x_{k+1}\right) \in X^{k+1}$ with $f\left(x_{1}, \ldots, x_{k}\right) \neq f\left(x_{2}, \ldots, x_{k+1}\right)$. Then, for any arbitrary points $x_{1}, \ldots, x_{k} \in X$, the sequence $\left\{x_{n}\right\}$ defined by (1) converges to $u$, and $u$ is a fixed point of $f$, that is, $u=f(u, \ldots, u)$. Moreover, if

$$
d(f(x, \ldots, x), f(y, \ldots, y)) \leq e^{-\tau} d(x, y),
$$

holds for all $x, y \in X$ with $x \neq y$, then $u$ is the unique fixed point of $f$.

Corollary 2.2. Let $(X, d)$ be a complete metric space, $k$ a positive integer and $f: X^{k} \rightarrow X$ a given mapping. Suppose that there exist $\lambda_{1}, \ldots, \lambda_{k}$ non-negative constants with $\lambda_{1}+\lambda_{2}+$ $\ldots+\lambda_{k}<1$ such that

(6) $d\left(f\left(x_{1}, \ldots, x_{k}\right), f\left(x_{2}, \ldots, x_{k+1}\right)\right) \leq \lambda_{1} d\left(x_{1}, x_{2}\right)+\lambda_{2} d\left(x_{2}, x_{3}\right)+\ldots+\lambda_{k} d\left(x_{k}, x_{k+1}\right)$, for all $\left(x_{1}, \ldots, x_{k+1}\right) \in X^{k+1}$ with $f\left(x_{1}, \ldots, x_{k}\right) \neq f\left(x_{2}, \ldots, x_{k+1}\right)$. Then, for any arbitrary points $x_{1}, \ldots, x_{k} \in X$, the sequence $\left\{x_{n}\right\}$ defined by (1) converges to $u$, where $u$ is the unique fixed point of $f$.

Proof. Clearly, condition (6) implies condition (5) with $\lambda=\lambda_{1}+\lambda_{2}+\ldots+\lambda_{k}$. Now, let $x, y \in X$ with $x \neq y$. From (6), we have

$$
\begin{aligned}
d(f(x, x, \ldots, x), f(y, y, \ldots, y)) \leq & d(f(x, \ldots, x), f(x, \ldots, x, y))+d(f(x, \ldots, x, y), f(x, \ldots, x, y, y)) \\
& +\ldots+d(f(x, y, \ldots, y), f(y, y, \ldots, y)) \\
\leq & \left(\lambda_{k}+\lambda_{k-1}+\ldots+\lambda_{1}\right) d(x, y)=\lambda d(x, y),
\end{aligned}
$$

where $\lambda=\lambda_{k}+\lambda_{k-1}+\ldots+\lambda_{1} \in(0,1)$. Hence all the hypotheses of Corollary ?? are satisfied and the result follows. 
If we take $F(\alpha)=-\frac{1}{\sqrt{\alpha}}$ for $\alpha \in \mathbb{R}^{+}$, then $F \in \digamma$ and we obtain the following nonlinear contraction that generalizes the contraction of type $d(f x, f y) \leq \alpha(d(x, y)) d(x, y)$ (see Boyd and Wong [6]).

Corollary 2.3. Let $(X, d)$ be a complete metric space, $k$ a positive integer and $f: X^{k} \rightarrow X$ a given mapping. Suppose that there exists $\tau>0$ such that

$d\left(f\left(x_{1}, \ldots, x_{k}\right), f\left(x_{2}, \ldots, x_{k+1}\right)\right) \leq \frac{1}{\left(1+\tau \sqrt{\max \left\{d\left(x_{i}, x_{i+1}\right): 1 \leq i \leq k\right\}}\right)^{2}} \max \left\{d\left(x_{i}, x_{i+1}\right): 1 \leq i \leq k\right\}$,

for all $\left(x_{1}, \ldots, x_{k+1}\right) \in X^{k+1}$ with $f\left(x_{1}, \ldots, x_{k}\right) \neq f\left(x_{2}, \ldots, x_{k+1}\right)$. Then, for any arbitrary points $x_{1}, \ldots, x_{k} \in X$, the sequence $\left\{x_{n}\right\}$ defined by (1.1) converges to $u$, that is, $u=$ $f(u, \ldots, u)$. Moreover, if

$$
d(f(x, \ldots, x), f(y, \ldots, y)) \leq \lambda d(x, y),
$$

holds for all $x, y \in X$ with $x \neq y$ where $\lambda \in[0,1)$, then $u$ is the unique fixed point of $f$.

Remark 2.1. (1) Theorem 2.1 extends and generalizes Theorem 1.2 in [7], and Theorem 1.1 in $[16]$.

(2) If $k=1$, Theorem 2.1 reduces to the Theorem 2.1 of Wardowski in [23].

(3) If $k=1$, Corollaries 2.2-?? reduces to Theorem 1 of Banach in [1].

(4) If $k=1$, Corollary ?? reduces to the Theorem of Boyd and Wong in [6].

\section{Global Attractivity Results for a Class of Matrix Difference Equations}

In this section, we investigate the global attractivity of the recursive sequence $\left\{X_{n}\right\} \subset$ $P(N)$ defined by

$$
X_{n+k}=Q+\frac{1}{k} \sum_{i=0}^{k-1} A^{*} \varphi\left(X_{n+i}\right) A, \quad n=1,2, \ldots,
$$

where $P(N)$ is the set of $N \times N$ Hermitian positive definite matrices, $k$ is a positive integer, $Q$ is an $N \times N$ Hermitian positive semidefinite matrix, $A$ is an $N \times N$ nonsingular matrix, $A^{*}$ is the conjugate transpose of $A$ and $\varphi: P(N) \rightarrow P(N)$.

At first, we recall some definitions and preliminary results.

Definition 3.1. Let $k$ be a positive integer, $M$ a nonempty set and $f: M^{k} \rightarrow M$. For given $x_{1}, x_{2}, \ldots, x_{k} \in M$, consider the recursive sequence $\left\{x_{n}\right\} \subset M$ defined by

$$
x_{n+k}=f\left(x_{n}, x_{n+1}, \ldots, x_{n+k-1}\right), \quad n=1,2, \ldots,
$$

The equilibrium point $\bar{x}$ of the equation (3.2) is the point that satisfies the condition:

$$
\bar{x}=f(\bar{x}, \ldots, \bar{x}) .
$$

Definition 3.2. Let $(M, d)$ be a metric space and $\bar{x}$ an equilibrium point of Equation (8). The equilibrium point $\bar{x}$ is called a global attractor if for all $x_{1}, x_{2}, \ldots, x_{k} \in M$, we have $d\left(x_{n}, \bar{x}\right) \rightarrow 0$ as $n \rightarrow \infty$. 
We denote by $P(N)$ (for $N \geq 2$ ), the open convex cone of all $N \times N$ Hermitian positive definite matrices. We endow $P(N)$ with the Thompson metric defined by

$$
A, B \in P(N), \quad d(A, B)=\max \{\ln M(A / B), \ln M(B / A)\},
$$

where $M(A / B)=\inf \{\theta>0: A \leq \theta B\}=\theta^{+}\left(B^{-1 / 2} A B^{-1 / 2}\right)$, the maximal eigenvalue of $B^{-1 / 2} A B^{-1 / 2}$. Here, $X \leq Y$ means that $Y-X$ is positive semidefinite and $X<Y$ means that $Y-X$ is positive definite. From Nussbaum [13], $P(N)$ is a complete metric space with respect to the Thompson metric $d$ and $d(A, B)=\left\|\ln \left(A^{-1 / 2} B A^{-1 / 2}\right)\right\|$, where $\|\cdot\|$ stands for the spectral norm. The Thompson metric exists on any open normal convex cones of real Banach spaces [13, 22]; in particular, the open convex cone of positive definite operators of a Hilbert space. Now we shortly introduce the elegant properties of the Thompson metric. It is invariant under the matrix inversion and congruence transformations, that is,

$$
d(A, B)=d\left(A^{-1}, B^{-1}\right)=d\left(U^{*} A U, U^{*} B U\right),
$$

for any nonsingular matrix $U$. The other useful result is the nonpositive curvature property of the Thompson metric

$$
d\left(X^{r}, Y^{r}\right) \leq r d(X, Y), \quad r \in[0,1] .
$$

According to (9) and (10), we have

$$
d\left(U^{*} X^{r} U, U^{*} Y^{r} U\right) \leq|r| d(X, Y), \quad r \in[-1,1] .
$$

Lemma 3.1. Lemma 3.3. For any $A, B, C, D \in P(N)$,

$$
d(A+B, C+D) \leq \max \{d(A, C), d(B, D)\} .
$$

Furthermore, for all positive semidefinite $A$ and $B, C \in P(N)$,

$$
d(A+B, A+C) \leq d(B, C) .
$$

Let $\varphi: P(N) \rightarrow P(N)$ be an $F$-contraction mapping with respect to the Thompson metric $d$. Let $Q$ be an $N \times N$ Hermitian positive semidefinite matrix $(Q \geq 0)$ and $A$ an $N \times N$ nonsingular matrix ( $A^{-1}$ exists). For a positive integer $k$, for given $X_{1}, X_{2}, \ldots, X_{k} \in$ $P(N)$, consider the sequence $\left\{X_{n}\right\} \subset P(N)$ defined by (7). Our main result in this section is the following.

Theorem 3.1. Equation (7) has a unique equilibrium point $\bar{X} \in P(N)$. Moreover, $\bar{X}$ is global attractor.

Proof. Define the mapping $f: P(N)^{k} \rightarrow P(N)$ by

$$
f\left(U_{1}, U_{2}, \ldots, U_{k}\right)=Q+\frac{1}{k}\left[A^{*} \varphi\left(U_{1}\right) A+A^{*} \varphi\left(U_{2}\right) A+\ldots+A^{*} \varphi\left(U_{k}\right) A\right],
$$

for all $U_{1}, U_{2}, \ldots, U_{k} \in P(N)$. 
Let $U_{1}, U_{2}, \ldots, U_{k+1} \in P(N)$. By using Lemma 3.3, we have

$$
\begin{aligned}
& d\left(f\left(U_{1}, U_{2}, \ldots, U_{k}\right), f\left(U_{2}, U_{3}, \ldots, U_{k+1}\right)\right. \\
& =d\left(Q+\frac{1}{k} \sum_{i=1}^{k} A^{*} \varphi\left(U_{i}\right) A, Q+\frac{1}{k} \sum_{j=2}^{k+1} A^{*} \varphi\left(U_{j}\right) A\right) \\
& \leq d\left(\frac{1}{k} \sum_{i=1}^{k} A^{*} \varphi\left(U_{i}\right) A, \frac{1}{k} \sum_{j=2}^{k+1} A^{*} \varphi\left(U_{j}\right) A\right) \\
& =d\left(\sum_{i=1}^{k}\left(\frac{1}{\sqrt{k}} A\right)^{*} \varphi\left(U_{i}\right)\left(\frac{1}{\sqrt{k}} A\right), \sum_{j=2}^{k+1}\left(\frac{1}{\sqrt{k}} A\right)^{*} \varphi\left(U_{j}\right)\left(\frac{1}{\sqrt{k}} A\right)\right) .
\end{aligned}
$$

Denote $V=\frac{1}{\sqrt{k}} A$. Then, using again Lemma 3.3, we have

$$
\begin{aligned}
& d\left(f\left(U_{1}, U_{2}, \ldots, U_{k}\right), f\left(U_{2}, U_{3}, \ldots, U_{k+1}\right)\right. \\
& \leq d\left(\sum_{i=1}^{k} V^{*} \varphi\left(U_{i}\right) V, \sum_{j=2}^{k+1} V^{*} \varphi\left(U_{j}\right) V\right) \\
& =d\left(V^{*} \varphi\left(U_{1}\right) V+V^{*} \varphi\left(U_{2}\right) V+\ldots+V^{*} \varphi\left(U_{k}\right) V, V^{*} \varphi\left(U_{2}\right) V+V^{*} \varphi\left(U_{3}\right) V+\ldots+V^{*} \varphi\left(U_{k+1}\right) V\right) \\
& \leq \max \left\{d\left(V^{*} \varphi\left(U_{1}\right) V, V^{*} \varphi\left(U_{2}\right) V\right), d\left(V^{*} \varphi\left(U_{2}\right) V, V^{*} \varphi\left(U_{3}\right) V\right), \ldots, d\left(V^{*} \varphi\left(U_{k}\right) V, V^{*} \varphi\left(U_{k+1}\right) V\right)\right\} \\
& =\max \left\{d\left(V^{*} \varphi\left(U_{i}\right) V, V^{*} \varphi\left(U_{i+1}\right) V\right): i=1,2, \ldots, k\right\} .
\end{aligned}
$$

Since $A$ is nonsingular, the matrix $V$ is also nonsingular. Using property (9), for all $i=1,2, \ldots, k$, we have

$$
d\left(V^{*} \varphi\left(U_{i}\right) V, V^{*} \varphi\left(U_{i+1}\right) V\right)=d\left(\varphi\left(U_{i}\right), \varphi\left(U_{i+1}\right)\right) .
$$

But $\varphi$ is an $F$-contraction. Then, for all $i=1,2, \ldots, k$, we have

$$
\tau+F\left(d\left(V^{*} \varphi\left(U_{i}\right) V, V^{*} \varphi\left(U_{i+1}\right) V\right)\right) \leq F\left(d\left(U_{i}, U_{i+1}\right)\right) .
$$

Thus, we have

$$
\tau+F\left(d\left(f\left(U_{1}, U_{2}, \ldots, U_{k}\right), f\left(U_{2}, U_{3}, \ldots, U_{k+1}\right)\right) \leq \max \left\{d\left(U_{i}, U_{i+1}\right): i=1,2, \ldots, k\right\}\right.
$$

for all $U_{1}, U_{2}, \ldots, U_{k+1} \in P(N)$.

Now, Applying Theorem 2.1, we obtain the existence of a global attractor equilibrium point $\bar{X} \in P(N)$. 
On the other hand, for $U, W \in P(N)$ such that $d(f(U, U, \ldots, U) \neq f(W, W, \ldots, W))$, we have

$$
\begin{aligned}
F(d(f(U, U, \ldots, U), f(W, W, \ldots, W))) & =F\left(d\left(Q+A^{*} \varphi(U) A, Q+A^{*} \varphi(W) A\right)\right) \\
& \leq F\left(d\left(A^{*} \varphi(U) A, A^{*} \varphi(W) A\right)\right) \\
& =F(d(\varphi(U), \varphi(W))) \\
& \leq F(d(U, W))-\tau,
\end{aligned}
$$

implies that

$$
\begin{aligned}
\tau+F(d(f(U, U, \ldots, U), f(W, W, \ldots, W))) & \leq \tau+F(d(\varphi(U), \varphi(W))) \\
& \leq F(d(U, W)) .
\end{aligned}
$$

Again, applying Theorem 2.1, we obtain the uniqueness of the equilibrium point.

Now, we present some examples and numerical experiments.

For a positive integer $k$, consider the sequence $\left\{X_{n}\right\} \subset P(N)$ defined by

$$
X_{n+k}=Q+\frac{1}{k} \sum_{i=0}^{k-1} A^{*} X_{n+i}^{\delta} A, \quad n=1,2, \ldots
$$

for given $X_{1}, X_{2}, \ldots, X_{k} \in P(N)$, where $|\delta| \in[0,1)$.

Corollary 3.1. Corollary 3.5. Equation (12) has a unique equilibrium point $\bar{X} \in P(N)$. Moreover, $\bar{X}$ is global attractor.

Proof. By using Properties (9) and (11), we show easily that $\varphi: P(N) \rightarrow P(N)$ defined by

$$
\varphi(X)=X^{\delta}, \quad \text { for all } X \in P(N)
$$

is $|\delta|$-contraction. Then, the result follows immediately from Theorem ??.

Remark 3.6. The equilibrium point $\bar{X} \in P(N)$ of Equation (12) is the unique positive definite solution to the nonlinear matrix equation

$$
\bar{X}=Q+A^{*} \bar{X}^{\delta} A .
$$

In the last few years there has been a constantly increasing interest in developing the theory and numerical approaches for positive definite solutions to the nonlinear matrix equation of the form (13) (see, for example, $[4,8,12]$ ).

As an example, we consider for given $X_{1}, X_{2} \in P(N)$, the recursive sequence $\left\{X_{n}\right\} \subset$ $P(N)$ given by

$$
X_{n+2}=Q+\frac{1}{2}\left(A^{*} X_{n}^{1 / 2} A+A^{*} X_{n+1}^{1 / 2} A\right), \quad n=1,2, \ldots
$$

From Corollary 3.1, Equation (14) has a unique equilibrium point $\bar{X} \in P(N)$, that is, the unique positive definite solution to

$$
\bar{X}=Q+A^{*} \bar{X}^{1 / 2} A
$$

To check our global attractivity result, we give the following numerical experiments. 
We take $N=3, Q$ and $A$ are given by

$$
Q=\left(\begin{array}{lll}
0.2 & 0.1 & 0.1 \\
0.1 & 0.2 & 0.1 \\
0.1 & 0.1 & 0.2
\end{array}\right), \quad A=\left(\begin{array}{lll}
1 & 2 & 3 \\
3 & 1 & 2 \\
2 & 3 & 1
\end{array}\right)
$$

For each iteration $i$, we consider the residual error $E(i)$ given by

$$
E(i)=\left\|X_{i}-\left(Q+A^{*} X_{i}^{1 / 2} A\right)\right\|,
$$

where $\|\cdot\|$ is the spectral norm. All programs are written in MATLAB version 7.1.

Let us take

$$
X_{1}=\left(\begin{array}{ccc}
2 & 1 & 0 \\
1 & 3 & 1 \\
0 & 1 & 4
\end{array}\right) \text { and } X_{2}=\left(\begin{array}{ccc}
5 & 5 & 1 \\
5 & 11 & 7 \\
1 & 7 & 17
\end{array}\right)
$$

then after 90 iterations of iterative method (14), we get the unique equilibrium point

$$
\bar{X} \approx X_{90}=\left(\begin{array}{lll}
438.4 & 429.2 & 429.2 \\
429.2 & 438.4 & 429.2 \\
429.2 & 429.2 & 438.4
\end{array}\right)
$$

and its residual error $E(90)=1.0503 e-013$.

For other initial points

$$
X_{1}=\left(\begin{array}{ccc}
120 & 7 & 7 \\
7 & 120 & 7 \\
7 & 7 & 120
\end{array}\right), \quad X_{2}=\left(\begin{array}{ccc}
1003 & 3 & 3 \\
3 & 2003 & 3 \\
3 & 3 & 3003
\end{array}\right)
$$

after 90 iterations, we get the unique equilibrium point $\bar{X}$ given by (15), and its residual error $E(90)=2.0196 e-013$.

Acknowledgment: The authors are grateful to the referees for their careful reading and critical remarks which undoubtedly helped us to improve the paper.

\section{References}

[1] S. Banach, Sur les opérations dans les ensembles abstraits et leur application aux équations intégrales, Fund. Math. 3 (1922), 133-181.

[2] V. Berinde and M. Păcurar, An iterative method for approximating fixed points of Prešić nonexpansive mappings, Rev. Anal. Numer. Theor. Approx. 38 (2) (2009), 144-153.

[3] V. Berinde and M. Păcurar, Two elementary applications of some Prešić type fixed point theorems, Creat. Math. Inform. 20 (1) (2011), 32-42.

[4] M. Berzig and B. Samet, Solving systems of nonlinear matrix equations involving Lipshitzian mappings, Fixed Point Theory Appl. 2011:89 (2011).

[5] Y.Z. Chen, A Prešić type contractive condition and its applications, Nonlinar Anal. 71 (2009), 20122017.

[6] D.W. Boyd and J.S.W. Wong, On nonlinear contractions, Proc. Am. Math. Soc. 20 (1969), 458-464.

[7] L.B. Ćirić and S.B. Prešić, On Prešić type generalization of the Banach contraction mapping principle, Acta Math. Univ. Comenianae. 76 (2) (2007), 143-147. 
[8] X. Duan, A. Liao and B. Tang, On the nonlinear matrix equation $X-\sum_{i=1}^{m} A_{i}^{*} X^{\delta_{i}} A_{i}=Q$, Linear Algebra Appl. 429 (2008), 110-121.

[9] H. H. Alsulami, E.Karapinar and H.Piri, Fixed Points of Modified F-Contractive Mappings in Complete Metric-Like Spaces,, Journal of Function Spaces, (2014) Article Id: 270971

[10] E. Karapinar, H. Piri and H. AlSulami, Fixed Points of Generalized F-Suzuki Type Contraction in Complete b-Metric Spaces," Discrete Dynamics in Nature and Society, 2015 (2015), Article ID 969726, 8 pages

[11] M.S. Khan, M. Berzig and B. Samet, Some convergence results for iterative sequences of Prešić type and applications, Adv. Difference Equ. 2012:38 (2012).

[12] Y. Lim, Solving the nonlinear matrix equation $X=Q+\sum_{i=1}^{m} A_{i}^{*} X^{\delta_{i}} A$ via a contraction principle, Linear Algebra Appl. 430 (2009), 1380-1383.

[13] R. Nussbaum, Hilbert's projective metric and iterated nonlinear maps, Mem. Amer. Math. Soc. 75 (391) (1988), 1-137.

[14] M. Păcurar, Approximating common fixed points of Presic-Kannan type operators by a multi-step iterative method An. stiint. Univ. Ovidius Constanţa Ser. Mat. 17 (1) (2009), 153-168.

[15] M. Pǎcurar, A multi-step iterative method for approximating fixed points of prešić-kannan operators, Acta Math. Univ. Comenianae. 79 (1) (2010), 77-88.

[16] S.B. Prešić, Sur une classe d'inéquations aux diffé rences finies et sur la convergence de certaines suites, Publ. Inst. Math. 5 (19) (1965), 75-78.

[17] S. Shukla, Prešić type results in 2-Banach spaces, Afr. Mat., (2013) DOI 10.1007/s13370-013-0174-2.

[18] S. Shukla and R. Sen, Set-valued Prešić -Reich type mappings in metric spaces, Revista de la Real Academia de Ciencias Exactas, Fisicas y Naturales. Serie A. Math. (2012). DOI 10.1007/s13398-0120114-2.

[19] S. Shukla, R. Sen and S. Radenović, Set-valued Prešić type contraction in metric spaces, An. Ştiinţ. Univ. Al. I. Cuza Iaşi. Mat. (N.S.) (To appear).

[20] S. Shukla and M. Abbas, Fixed point results of cyclic contractions in product spaces, Carpathian J. Math., In press (2014).

[21] S. Shukla, S. Radojević, Z. A. Veljković and S. Radenović, Some coincidence and common fixed point theorems for ordered Prešić -Reich type contractions, J.Inequal. Appl. 2013, 2013:520 DOI 10.1186/1029-242X-2013-520.

[22] A. Thompson, On certain contraction mappings in a partially ordered vector space, Proc. Am. Math. Soc. 14 (1963), 438-443.

[23] D. Wardowski, Fixed points of new type of contractive mappings in complete metric spaces. Fixed Point Theory Appl., 2012:94 (2012), 6 pages. 